\title{
Impact of COVID-19 on General Surgical Practice in India
}

\section{Amrit Manik Nasta ${ }^{1}$ (D) Ramen Goel $^{1}$ (D) $\cdot$ Manickavasagam Kanagavel $^{2}$ (D) Sundaram Easwaramoorthy $^{3}$}

Received: 13 May 2020 / Accepted: 29 May 2020 / Published online: 26 June 2020

(C) Association of Surgeons of India 2020

\begin{abstract}
The coronavirus disease 2019 (COVID-19) pandemic is a global health crisis, and surgeons are at increased occupational risk of contracting COVID-19. The impact of the disease on prevalent general surgical practice is uncertain and continues to evolve. The study aimed to study the impact of COVID-19 on general surgical practice in India and the future implications of the pandemic. A survey questionnaire was designed and electronically circulated 1 month after India entered a national lockdown during COVID19 pandemic, amongst members of Indian Association of Gastro-intestinal Endo-surgeons (IAGES), a surgical association with nearly eight thousand members from across the country. Survey questions pertaining to pre-COVID era surgical practices, impact on current practice, and financial implications were asked. Responses were collected and statistically analyzed. One hundred fifty-three surgeons completed the survey, of which only $9.2 \%$ were women. Majority $(41 \%)$ were into practice for more than 20 years; $36.6 \%$ were into private practice at multiple hospitals (free-lancers). Amongst the respondents, $41.8 \%$ had mainly laparoscopic practice with mean outpatient consultation of 26 patients/day and elective surgeries of 43 cases/month prior to lockdown. Post-lockdown, daily outpatient consults reduced to 4 patients per day, and $77 \%$ had not performed a single elective procedure. Hydroxychloroquine (HCQ) chemoprophylaxis was reported by $52 \%$ surgeons. Personal protective equipment (PPE) was used by $52 \%$ for all cases, while $71.5 \%$ stated there are insufficient guidelines for future surgical practice in terms of safety. A drop of more than $75 \%$ of their monthly income was experienced by $52 \%$ surgeons, while $22 \%$ faced $50-75 \%$ reduction. One third (33\%) of respondents own a hospital and are expecting a monthly financial liability of 2.25 million rupees (nearly 30,000 US dollars). COVID-19 has led to a drastic reduction in outpatient and elective surgical practices. There is a definite need for guidelines regarding safety for future surgical practices and solutions to overcome the financial liabilities in the near future.
\end{abstract}

Keywords COVID-19 $\cdot$ Surgery $\cdot$ Laparoscopy $\cdot$ Safety $\cdot$ Financial impact

Amrit Manik Nasta

amritnasta@hotmail.com

Ramen Goel

ramengoel@gmail.com

Manickavasagam Kanagavel

drmkvel@yahoo.com

Sundaram Easwaramoorthy

easwarmoorthy2007@rediffmail.com

1 Center of Bariatric \& Metabolic Surgery, Wockhardt Hospitals, Mumbai, India

2 Department of General, Gastrointestinal and Minimal Access Surgery, St Isabel's Hospital, 49, Oliver Road, Chennai 600 004, India

3 Department of Minimal Access Surgery, Poondurai Main Road, Kollampalayam, Erode 638002, India

\section{Introduction}

The novel coronavirus was declared a public health emergency of international concern (PHEIC) by the World Health Organization (WHO) on January 30, 2020 [1]. In the first week on March, an unexpectedly high number of cases were detected worldwide and coronavirus disease 2019 (COVID-19) was declared a pandemic on March 11, 2020 [2].The Indian government announced a countrywide lockdown for 3 weeks starting at midnight on March 24 to slow the spread of COVID-19 as the number of people testing positive in the country reached 563 [3]. However, this lockdown was further extended till May 3, 2020. One month into lockdown, outpatient clinics and elective surgeries were likely to have taken a beating. Majority of hospital resources were directed towards availing masks and personal protective equipment (PPE), minimizing staff movements and suspension of all elective work. 
Aims The study aimed to study the impact of COVID-19 on general surgical practice in India and the future implications of the pandemic.

Methods This survey was conducted at a tertiary-care hospital. The survey questionnaire was designed and electronically circulated 1 month after India entered a national lockdown, amongst members of Indian Association of Gastro-intestinal Endo-surgeons (IAGES), nearly 8000 member strong association with surgeons having interest in general and laparoscopic surgery. Survey questions pertaining to pre-COVID era surgical practices, impact on current practice, and financial implications were asked. Responses were collected, and Chi square test was used for statistical analysis.

The self-administered questionnaire consisted of twentyone questions with five part socio-demographic questions, questions on outpatient and surgical (emergency/elective) numbers in pre-lockdown and post-lockdown period, safety practices, and financial impact in the current period.

Results One hundred and fifty-three surgeons from across the country completed the survey, of which only $9.2 \%$ were women. Amongst the respondents, $41.2 \%$ surgeons were more than 20 years into practice, $34 \%$ for 10 to 20 years, and $22.2 \% 4$ to 10 years after completing their specialty degree. For place of practice, $36.6 \%$ were into private practice at multiple hospitals (free-lancers), $29.4 \%$ were full timers at a single corporate hospital, and $13.1 \%$ were full timers at government hospitals.

Prior to Lockdown Amongst the respondents, $41 \%$ had mainly laparoscopic practice and $41.7 \%$ had equal proportion of laparoscopic and open surgery (Fig. 1). Surgeons reported a mean outpatient consultation of 26 patients/day and elective surgeries 42 cases/month prior to lockdown.

Post-lockdown Since the beginning of lockdown, 36.5\% reported to have completely stopped outpatient services, $63.5 \%$

\section{Types of Surgical practice}

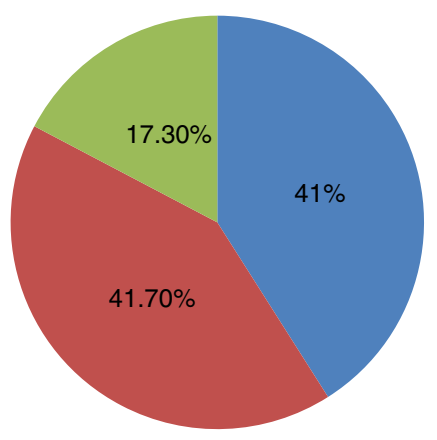

Fig. 1 Distribution of open and laparoscopic surgical practice prior to lockdown
- Laparoscopic

Equal proportion

- Open surgeons had a reduction in their services, and $50 \%$ reported to have started online consultations. Amongst those continuing consultations, average daily consults reduced to 4 patients per day.

All elective surgical work was stopped by $93.3 \%$, while $5.2 \%$ had scaled down elective surgeries. No elective procedures were performed by $77 \%$, while $16 \%$ performed less than 5 surgeries during the lockdown period. No emergency surgeries were performed by $9 \%$ surgeons, and $42.5 \%$ had reduced emergency services where feasible. Average elective and emergency surgeries performed in the month of lockdown were merely one and five in number, respectively (Fig. 2). The reduction in OPD, elective, and emergency surgical practice was statistically significant $(p<0.05)$.

Safety Practices Hydroxychloroquine (HCQ) was taken by $52 \%$ surgeons for chemoprophylaxis. It was felt by $93 \%$ surgeons that laparoscopic surgery and use of energy sources increased the risk of aerosol spread of the virus. For safe surgical practice, $56.9 \%$ of surgeons said they would use PPE in all cases, $38.5 \%$ would prefer open surgery, and $33.1 \%$ would use filters for de-sufflation. More evidence was sought by $71.5 \%$ of surgeons to understand safety practices in future (Fig. 3).

Half the surgeons (50.8\%) claimed COVID-19 rapid antigen test had false negative rate of up to $30 \%$, while $43.1 \%$ claimed high false negative antibody rates are seen in first week of infection. When asked to select top three areas where adequate information is lacking on COVID-19 for surgeons, most surgeons selected future of surgery in COVID era $(77.7 \%)$, safety in laparoscopy $(69.2 \%)$, and safety of staff (59.2\%) (Fig. 4). For sources of COVID-19 information, $76.1 \%$ utilized internet as a medium, 5.9\% utilized television, while $15.4 \%$ utilized directives given on government/national body sources.

Financial Impact A drop of more than $75 \%$ of their monthly income was experienced by $52 \%$ surgeons, while $22 \%$ faced $50-75 \%$ reduction. Subgroup analysis revealed surgeons working in private hospitals had significantly $(p=0.000)$ greater reduction in income, compared to surgeon in government set up (Fig. 5). One third (33\%) of respondents owned a hospital and were expecting a monthly financial liability of 2.25 million rupees (30,000 US dollars).

\section{Discussion}

COVID-19 has led to significant worldwide change in surgical practice. Our survey showed that practice of majority of surgeons in India has been drastically affected by the COVID19 pandemic. As of April 24, 2020 at 9 a.m., over 23,000 cases were confirmed positive cases in India [4]. 
Fig. 2 Comparison of daily outpatient consultations (OPD) and monthly elective and emergency surgeries prior and postlockdown

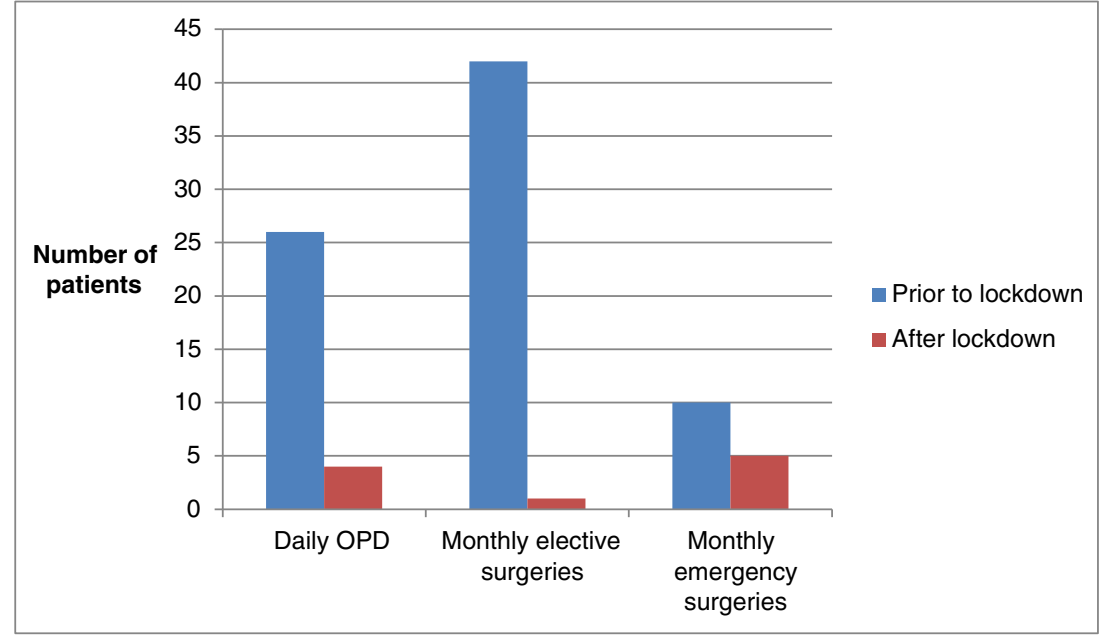

Outpatient consultations and non-emergency surgeries have declined from over 20 cases/day and 40 surgeries/ month to almost zero cases in this lockdown period. Spinelli [5] reported that most outpatient clinics were suspended in Italy, and scheduled patients are called beforehand by hospital administration, asking for specific symptoms in the previous 2 weeks (for example, fever or cough), or direct exposure to COVID-19-positive individuals. In such cases, the patient was asked not to come to the hospital and the visit was postponed. A survey on ophthalmology practice in India by Nair et al. [6] showed the $72.5 \%$ of the practicing ophthalmologists in India were in total lockdown. While the clinicians themselves may be available, the unavailability of managerial, administrative, nursing, and other support staff may pose logistical and operational challenges in running a health care facility during COVID-19 pandemic.

Surgeons are at dual risk of exposure in their practice, namely, both from the outpatient clinics and operating room. Our survey showed that nearly $50 \%$ surgeons have taken
HCQ as chemoprophylaxis. A systematic review by Shah et al. [7] showed an absence of robust in vivo evidence to support the role of HCQ in prevention of COVID-19. But the National Task Force for COVID-19 constituted by Indian Council of Medical Research (ICMR) on March 22, 2020, recommended HCQ for prophylactic use in asymptomatic healthcare workers (HCWs) involved in care of suspected or confirmed patients of COVID-19 (https://www.mohfw. gov.in/pdf/AdvisoryontheuseofHydroxychloroqu inasprophylaxisforSARSCoV2infection.pdf). When HCQ administration is considered for a COVID-19 patient or suspect, efforts should be made to advise high-risk individuals to have a baseline ECG recording [8].

After the pandemic is under control, it is unclear on the extent of precautions that would be required to be followed by surgical and operating room personnel. When asked about future concerns, most surgeons mentioned regarding the future of surgical practice in COVID era (78\%), safety in laparoscopy (69\%), and safety of staff (59\%). This mandates the
Fig. 3 Safety measures to be adopted for future surgeries

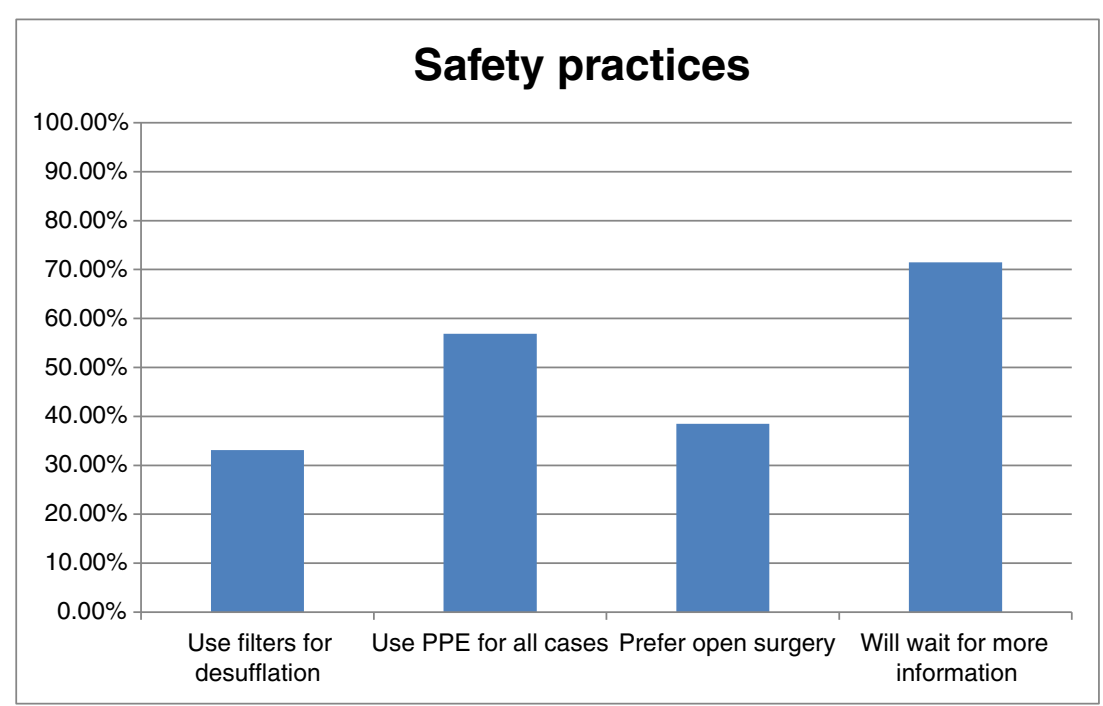


Fig. 4 Top three areas where information is lacking for surgery

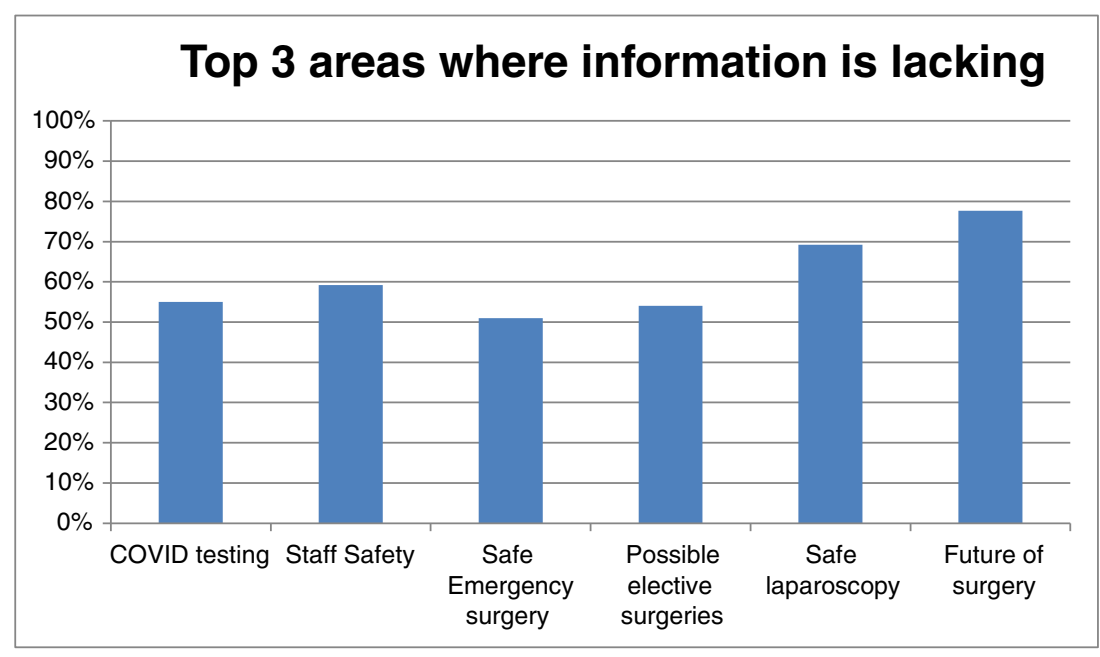

need for national and international guidelines for addressing these issues. Stahel [9] has provided an algorithm based on elective surgical indications and predicted proper perioperative utilization of critical resources, including the consideration for intraoperative/postoperative blood product transfusions, estimated postoperative hospital length of stay, and the expected requirement for prolonged ventilation and need for postoperative ICU admission.

Prior to COVID-19 lockdown in India, 42.2\% surgeons in our survey mainly did laparoscopic practice. According to a study by Tuech et al. [10], caution is required when performing laparoscopy because of the risk of aerosol release and subsequent risk of exposure to operating room personnel. The main risk comes from the possible presence of pathogens in the peritoneal cavity. The aerosol released into the theater during surgery from ports or after the operation (de-sufflation of abdomen) may contaminate personnel and equipment and surfaces in the room via airborne particles [10]. In our survey, 56.9\% claimed they would use PPE in all cases, $38.5 \%$ would prefer open surgery, and $33.1 \%$ would use filters for de-sufflation, while
71.5\% stated more evidence was required. Despite guidelines coming from various national and international surgical societies, there is still a major uncertainty about what safe practices are to be adopted.

Our survey reported that $77 \%$ of surgeons relied on internet as a premium source of COVID-19 information. Currently, the vast diversity of information available through the Internet, including unverified malicious information, can spread quickly and can misguide healthcare workers (HCWs). Health authorities and scientists have warned that widespread misinformation about COVID-19 is a serious concern causing xenophobia worldwide [11]. Another concern on surgical practice is the economic impact of the pandemic. Our survey reported $52 \%$ surgeons experienced a $75 \%$ or more reduction in their income, and the duration of this scenario is uncertain. In a recent survey conducted by Irish Dental Association (IDA), which involved 369 dentists, it was reported that one-fifth of the dentists have closed their practices (temporarily or permanently). In addition, around threefourth of the participants are expecting a financial loss of over $70 \%$ amid COVID-19 outbreak [12].
Fig. 5 Comparison of financial losses in surgeons working in private versus government setup

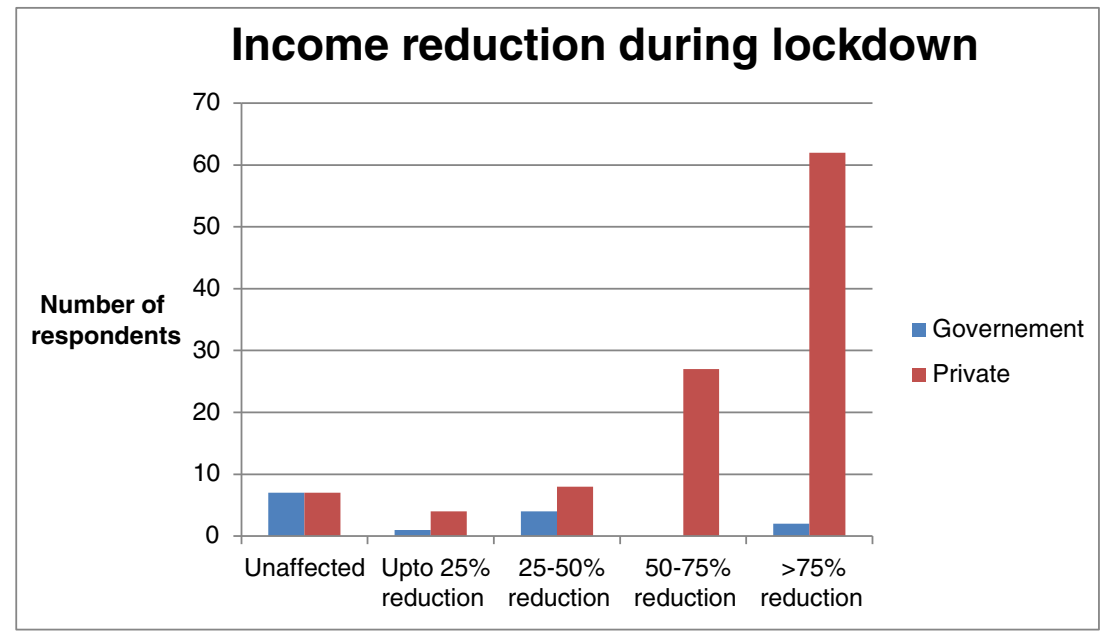


A limitation of the study was that COVID has affected different cities and states in India with variable intensity; hence, the perceptions of the responders will be limited.

\section{Conclusion}

Our survey highlights the need for more information on future of surgical practices, to make surgery safe in the times of the pandemic. The existing lockdown has had a major impact on routine surgical practice and will require dedicated efforts for resumption of "new normal" in future of laparoscopic surgery.

\section{Compliance with Ethical Standards}

Conflict of Interest The authors declare that they have no conflict of interest.

\section{Ethical Approval N/A}

Consent to Participate N/A

Consent for Publication N/A

\section{References}

1. Eurosurveillance Editorial Team (2020) Note from the editors: World Health Organization declares novel coronavirus (2019$\mathrm{nCoV}$ ) sixth public health emergency of international concern. Euro Surveill 25(5):200131e

2. WHO Director-General's opening remarks at the media briefing on COVID-19 - 11 March 2020 (2020) Accessed: Mar 19, 2020: https://www.who.int/dg/speeches/detail/who-director-general-sopening-remarks-at-the-media-briefing-on-covid-19\%2D\%2D-11March-2020
3. Pulla P (2020) Covid-19: India imposes lockdown for 21 days and cases rise. BMJ 368:m1251

4. ICMR testing update $24^{\text {th }}$ April, 2020. https://icmr.nic.in/sites/ default/files/whats_new/ICMR_testing_update_24Apr2020_ 9AM IST.pdf

5. Spinelli A, Pellino G (2020) COVID-19 pandemic: perspectives on an unfolding crisis. Published online in Wiley Online Library (www.bjs.co.uk). https://doi.org/10.1002/bjs.11627

6. Nair AG, Gandhi RA, Natarajan S (2020) Effect of COVID-19 related lockdown on ophthalmic practice and patient care in India: results of a survey. Indian J Ophthalmol 68:725-730

7. Shah S, Sad S, Jain A, Misra DP, Negi VS (2020) A systematic review of the prophylactic role of chloroquine and hydroxychloroquine in coronavirus disease-19 (COVID-19). Int J Rheum Dis 23:613-619. https://doi.org/10.1111/1756-185X.13842

8. Kapoor A, Pandurangi U, Arora V, Gupta A, Jaswal A, Nabar A, Naik A, Naik N, Namboodiri N, Vora A, Yadav R, Saxena A (2020, ISSN 0972-6292) Cardiovascular risks of hydroxychloroquine in treatment and prophylaxis of COVID-19 patients: a scientific statement from the Indian Heart Rhythm Society. Indian Pacing Electrophysiol J 20:117-120. https://doi. org/10.1016/j.ipej.2020.04.003

9. Stahel P (2020) How to risk-stratify elective surgery during the COVID-19 pandemic? Patient Saf Surg 14:8. https://doi.org/10. 1186/s13037-020-00235-9

10. Tuech J, Gangloff A, Di Fiore F, Michel P, Brigand C, Slime K, Pocard M, Schwarz L (2020) Strategy for the practice of digestive and oncological surgery during the Covid-19 epidemic. J Visceral Surg. https://doi.org/10.1016/j.jviscsurg.2020.03.008

11. Lai CC, Shih TP, Ko WC, Tang HJ, Hsueh PR (2020) Severe acute respiratory syndrome coronavirus 2 (SARS-CoV-2) and corona virus disease-2019 (COVID-19): the epidemic and the challenges. Int J Antimicrob Agent:105924. https://doi.org/10.1016/j.ijantimicag. 2020.105924

12. IDA News. Scale of dental collapse highlighted in survey. Available: http://irishdentalassociation.newsweaver.com/ newsletter $/ 13 \mathrm{ctcsav} 7 \mathrm{cz} 1$ cys $22 \mathrm{xtzj} 2 ? \mathrm{a}=1 \& \mathrm{p}=56657247 \& \mathrm{t}=$ 22322575

Publisher's Note Springer Nature remains neutral with regard to jurisdictional claims in published maps and institutional affiliations. 